\title{
A Laboratory Method for Obtaining two Degrees of Freedom Gyroscopic Stabilizer Transfer Function
}

\author{
Mohammad Sadegh Mirzajani Darestani ${ }^{1}$, Seyed Zeynolabedin Moussavi ${ }^{2}$, Parviz Amiri ${ }^{2}$ \\ ${ }^{1}$ Electrical Engineering Department, Islamic Azad university, Arak branch \\ Arak, Iran \\ smoussavi@srttu.edu \\ ${ }^{2}$ Faculty of Electrical Engineering, Shahid Rajaee Teacher Training University \\ Lavizan, Tehran, Iran \\ pamiri@srttu.edu
}

\begin{abstract}
Obtaining transfer function of electrical, mechanical, etc. systems can provide this possibility for the researchers to investigate the behaviours of desired systems based on different inputs in various working circumstances without need to laboratory equipment which it results in lower consumption of time and expense. The aim of current research is obtaining the existing gyroscopic stabilizer transfer function. The way we used in this article is the newest laboratory way for obtaining transfer function of gyroscopic stabilizers. This aim is achieved by using laboratory equipment such as a two degrees of freedom gyro stabilized platform that an imaging system is installed on it as the load, target simulator table with one degree of freedom, and electronic conversion board of RS488 to RS232 serial communication standard, etc. An arbitrary input is introduced to under test system and the system behaviour toward the introduced input is saved and finally the transfer function of existing two degrees of freedom gyro stabilized platform is obtained using system identification toolbox in MATLAB. At the end of this article, the step response of transfer function obtained through the desired experiment in the laboratory compared with the step response obtained through simulations that a $\% 10$ difference between them is observed.
\end{abstract}

Keywords: Gyro stabilized platform, Gyroscope, Transfer function, Degree of freedom

\section{Introduction}

There are different trade and industrial systems in use today where two degrees of freedom platforms are utilized for imaging. Such systems must be so stabilized during identifying and tracking as well as imaging the target that image resolution is not lost as a result of constant vibration and shaking. Two degrees of freedom gyro stabilized platforms are usually mounted on the nose of a guided device and can search the space within their field of view (FOV) to find the target and imaging it while maintaining their stability. Like all other control systems, the desired output, i.e., the platform angle is compared with the reference input, i.e., the imaging system line of sight (LOS) angle, and, subsequently, a compensator is issued the proper torque command based on the error signal from the error signal. The criteria for the final performance of the gyroscopic stabilizer which carries the imaging system are quality and resolution of the image generated by the optical sensor in spite of target and carrier motion. Instability reduction is generally considered as a suitable criterion for determining the proper performance of the stabilizer. In [1] and [3], the structure and operation of inertial stabilized platforms were studied. In these articles, the equations of motion related to these platforms were also fully discussed. Researchers in [2] specifically studied the direct and indirect stabilization of imaging system in two axis platforms and the performance of these two methods were also discussed and compared to each other. Modelling and design of a controller for two axis inertial stabilized platforms were addressed in [4]. In [9], the methods used for stabilizing electro-optical tracing/tracking platforms were studied. This article also discussed ways of controlling the platform in the tracking mode based on fuzzy control methods.

The transfer function of a system, the ratio of output to input of the system, can be obtained through different theoretical methods as well as practical experiments. 
As mentioned above, this research is based on a two degrees of freedom gyro stabilized platform. Therefore, knowledge of the transfer function of this system would help us evaluate the performance and behaviour of this platform under different ambient and working conditions.

One common approach for obtaining the transfer function of a system is injecting a signal to the system input and storing the corresponding output, thus obtaining the transfer function (the ratio of output to input). In the present study, we obtain the transfer function of a two degrees of freedom gyro stabilized platform through conducting practical tests. We assume that the platform is only a "black box" about the technical and functional components of which we have no information. Through giving a specific input to the system, we obtain the corresponding output and then use the system identification toolbox in MATLAB to determine the transfer function of the platform and determine some of its functional characteristics.

Next sections of this article are as follow: In section 2, we will get familiar with structure and working procedure of two degrees of freedom gyro stabilized platform. Section 3 was assigned to the explanation of proposed method in this article. Section 4 discusses the comparison of step responses obtained in laboratory and MATLAB/SIMULINK. Section 5 is the conclusion part of the article.

\section{Structure and Functioning of Gyroscopic Stabilized Two Degrees of Freedom Platform}

A very common application of two degrees of freedom gyro stabilized systems is the line of sight stabilization of a camera or imaging system. A line of sight stabilization system is a system which maintains the stability of the line of sight of an electro-optical sensor under external disturbance (movement of the supporting base, etc.) [1]. Under such conditions, when a vehicle rotates about its axes, its line of sight must remain constant relative to the inertial reference frame so that image resolution can be preserved in spite of successive vibration and shaking of the base.

The studied system is a two degrees of freedom gyro stabilized platform which can rotate at an arbitrary angular velocity in both clock wise and counter clock wise directions, and can be stopped at any specified arbitrary angle. This platform is expected to rotate the camera at constant angular velocity on either side of its movable axes. To this end, it is necessary to deploy a precise speed sensor inside a speed control loop with an appropriate PID controller.

The block diagram in Fig. 1 shows the elements of the two degrees of freedom gyro stabilized platform along both its axes. As shown in this block diagram, the torque motor and the potentiometer are positioned on either side of the gimbal and lie along either axis. Motion of the frames is generated via two high torque direct current (DC) motors. Two potentiometers are used to read the camera position relative to the fixed platform. A rate gyroscope inside the gimbal senses the angular velocities about axes parallel with its own in the horizontal and vertical directions.

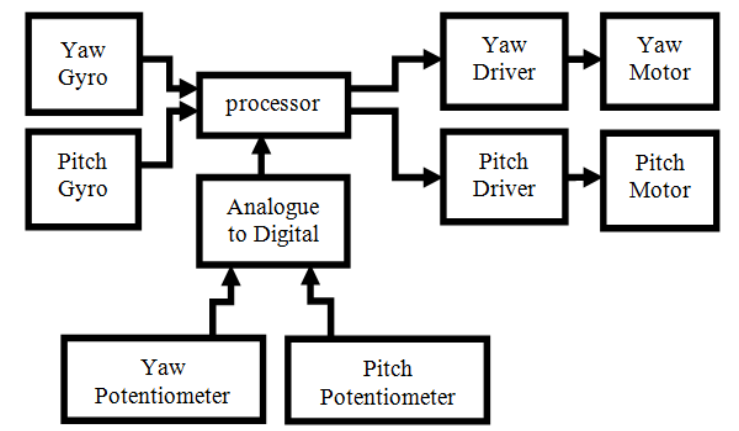

Fig. 1: Block diagram showing the components and internal relations of the two degrees of freedom gyro stabilized platform.

This type of platforms generally includes a body and a gimbal consisting of two nested frames providing separate movements for the camera along horizontal and vertical directions. These movements can be independently controlled. Thus, displacement along one axis has no effect on the other axis. The outer and the inner frames provide motion along horizontal and vertical axes respectively [2]. Figure 2 shows the general method of controlling the system along 
each axis. The system needs to move smoothly and continuously without any redundant or jerky movements so that the camera can perform correctly. Appropriate position and velocity measurements of the camera are made via sensors. The output of these sensors is converted into command signals for the motor actuating the respective axis, and applied to this motor through amplifiers particularly designed for this purpose. The high precision used for the related movements and the control system require that force transmission and measurement system also act precisely [3]. Thus, a directly coupled power transfer system can be implemented.

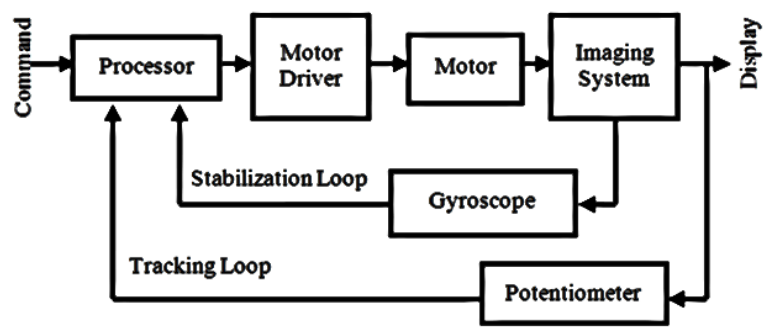

Fig. 2: Block diagram for the control loops of a two degrees of freedom gyro stabilized platform.

Two potentiometers are used to read the camera position relative to the fixed platform. These are connected via gears to the pitch and yaw axes. The potentiometer resistance changes based on the positions of the gears and the movement of each axis. Each axis position can be determined by reading the voltage at the middle pin of the potentiometer. Potentiometer voltages can be converted into digital signals via the analogue to digital circuit and made available to the processor.

Figure 3 shows the angles defined for describing the relative angular positions of the imaging target, the two degrees of freedom gyro stabilized platform, and the planar motion of the carrier axis of the two degrees of freedom gyro stabilized platform

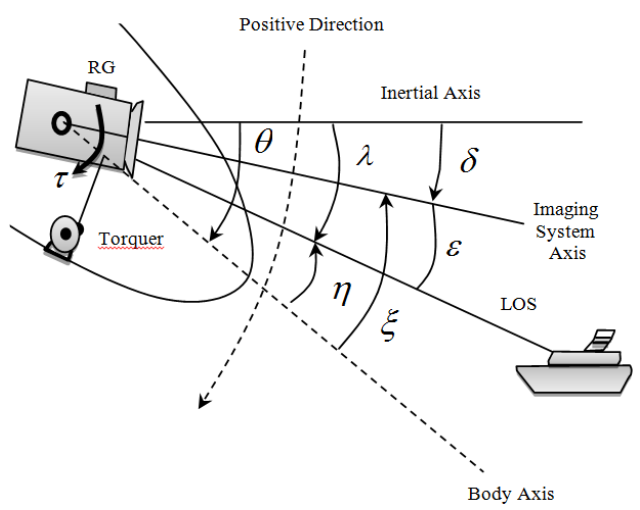

Fig. 3: Target tracking two dimensional Geometry.

The angles shown in Fig. 3 are:

$\theta$ : Angle between the inertial axis and carrier body axis

$\lambda$ : Angle between the inertial and axis imaging system line of sight

$\eta$ : angle between the carrier body axis and imaging system line of sight

$\varepsilon$ : Angle between the imaging system line of sight and imaging system axis

$\delta$ : Angle between the inertial axis and imaging system axis

$\xi$ : Angle between the carrier body and imaging system axis

LOS: Imaging system line of sight 
Thus, two control loops acting in opposite directions are created for searching and tracking the target. The purpose of the tracking loop is to move the two degrees of freedom gyro stabilized platform and the purpose of the stabilizing loop is to fix the line of sight relative to the inertial frame [4]. Since the frequency domains for tracking and noise reduction are different, these two loops shall not interfere with each other's operation. Figure 4 shows the control loops for the two degrees of freedom gyro stabilized platform.

As shown in Fig. 4, the potentiometer used in the platform measures the angle between the imaging system axis used in the stabilized platform and the platform body $(\delta)$ and feeds it as input to the system. Mechanical noise $(\theta)$ due to carrier movement is also included in the imaging system angle [5]. The angle measured by the potentiometer $\left(\zeta_{M}\right)$ is compared at the input of tracking loop with a reference input $\left(\zeta_{\mathrm{CM}}\right)$. The difference between these angles $(\mathrm{e})$ is entered into the compensator in the tracking loop. This compensator sends to the stabilizing loop an angular velocity command $\left(\omega_{\mathrm{CM}}\right)$ for compensating the detected difference. Once this angular velocity command has been entered into the stabilizing loop, the torque motors guide the imaging system at the maximum allowable angular velocity $(\dot{\delta})$ towards the desired angular position [6].

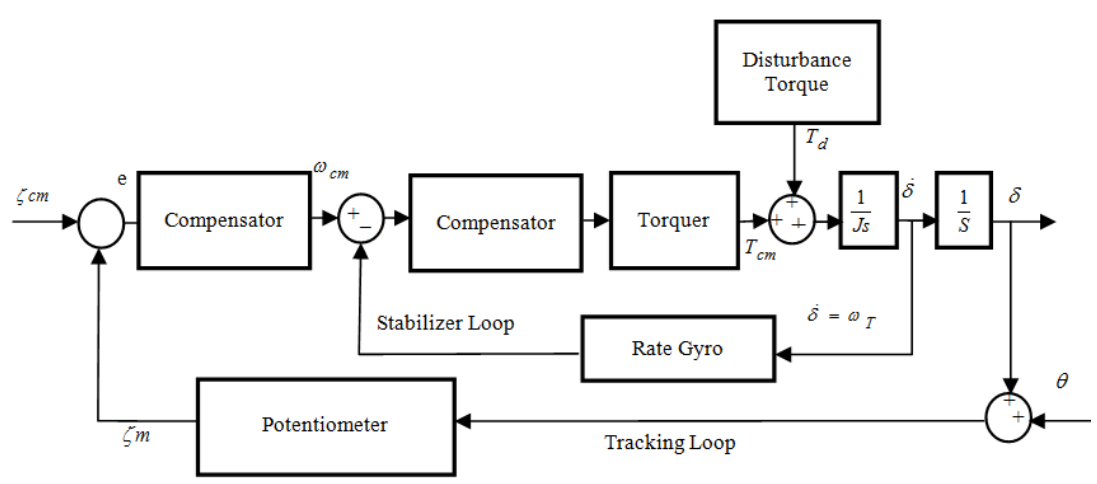

Fig. 4: Block diagram for control loops of the two degrees of freedom gyro stabilized platform [7].

The gyroscopic stabilizer used in this study has two working modes, namely, search mode and track mode. The track mode is used to obtain the platform transfer function. In the track mode, the imaging system is locked on the target obtained through image processing algorithms during the search mode. In the track mode, the existing gyroscopic stabilizer is constantly trying to maintain the target near the field of view centre in the imaging system.

\section{Proposed Method}

In this method, to get the transfer function of two degrees of freedom gyro stabilized platform some essential equipment such as two degrees of freedom gyro stabilized platform, One degree of freedom target simulator, laptop, required cables, fixtures, and a serial board is needed. A picture of the one degree of freedom target simulator is shown in Fig. 5. 


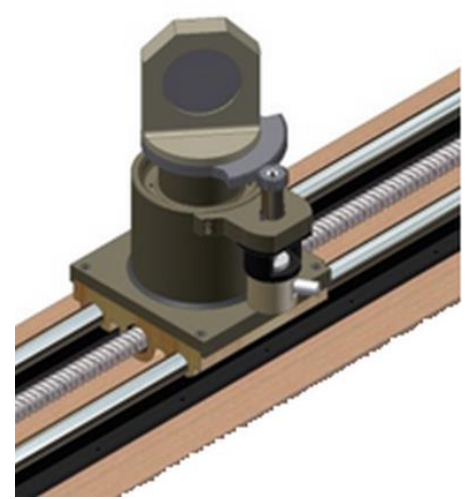

Fig. 5: A view of the one degree of freedom target simulator table.

As mentioned in the section 2 of this article, an infrared imaging system is mounted on the two degrees of freedom platform. Thus, these types of platforms (with infrared imaging system) are also used for searching and tracking targets which emit infrared waves. In fact, the one degree of freedom target simulator acts as a moving infrared target [8]. Therefore, the two degrees of freedom gyro stabilized platform can implement image processing algorithms to search for and track this infrared target. The target simulator is capable of moving at the desired frequency and angular velocity about the center of the target simulator. To simulate the infrared target, a resistor is used the ends of which are connected to a power supply during the test. After this resistor has been heated, the two degrees of freedom platform with infrared imaging system can identify and subsequently track it as an infrared target.

The purpose of this test is to obtain the transfer function of the two degrees of freedom gyro stabilized platform through injecting the desired signal to the input of this system and storing the corresponding output. To realize this, first the resistor (which acts as the infrared target for the two degrees of freedom gyro stabilized platform) must be heated through connecting it to the power supply. Then, the two degrees of freedom gyro stabilized platform must be positioned in front of the target simulator in a way that the field of view (FOV) of the two degrees of freedom gyro stabilized platform can completely cover the motion interval of target simulator.

The gyroscopic stabilizer has a serial output port for online transmission of some of the important functional parameters of platform and signals in accordance with the RS488 serial communication standard as the platform moves. In fact, the data sent through this serial port to the output contain several columns each of which describes the status of one important functional parameter of the two degrees of freedom gyro stabilized platform (such as signals showing the platform movement for searching in yaw axis or pitch axis).

MATLAB was used to store the output data from the RS422 serial port of platform. The output data from the serial port were converted into the RS232 standard data and transferred to the serial port of the computer.

An $\mathrm{m}$-file was created to store the data transferred to the serial port of the computer. Upon running the $\mathrm{m}$-file, all the data related to functional parameters of the existing platform were stored.

Another m-file was deployed to classify the stored data into separate signals under the desired topics for the purpose of individually analyzing each signal. Upon executing this $\mathrm{m}$-file, each of the existing signals was separately classified under the desired name in the Workspace window.

One input which can be used for identifying the system for the purpose of obtaining its transfer function is the command issued by the image processing algorithm to the two degrees of freedom gyro stabilized platform for searching about the yaw axis. Therefore, the output corresponding to this input would be a signal received from the gyroscope mounted in the direction of yaw rotation in response to the issued command. Thus, by storing both these signals, we can obtain the transfer function of the existing two degrees of freedom gyro stabilized platform using the system identification toolbox in MATLAB. 
To start the test, we first adjust the two degrees of freedom gyro stabilized platform in front of the one degree of freedom target simulator in a way that the field of view of the two degrees of freedom gyro stabilized platform cover the target simulator's motion interval and then connect the resistor (which acts as an infrared target) to the power supply. Then, we rotate the target simulator at a specific frequency and angular range about its center. Subsequently, the stabilizer is set at the track mode so that it can lock on the target and keep the target near the center of the imaging system field of view. As such, the two degrees of freedom gyro stabilized platform can follow the target as the target rotates about the yaw axis. By running the first m-file in MATLAB, we can save the output signal of the existing platform's serial port (which actually includes the command signal sent from the image processing algorithm to the existing platform as well as the existing platform reaction to the transmitted input signal).

Figure 6 shows the command sent from the image processing algorithm to the studied two degrees of freedom gyro stabilized platform for searching about the yaw axis as well as the stored output signal obtained from the gyroscope mounted on the yaw axis in response to the command sent by the image processing algorithm to the existing gyroscopic stabilizer.

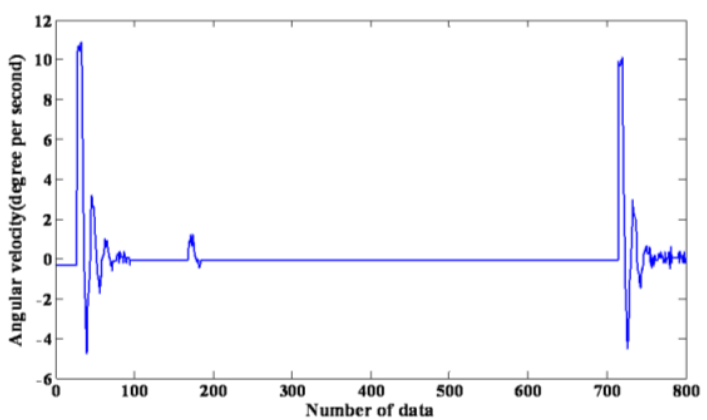

(a)

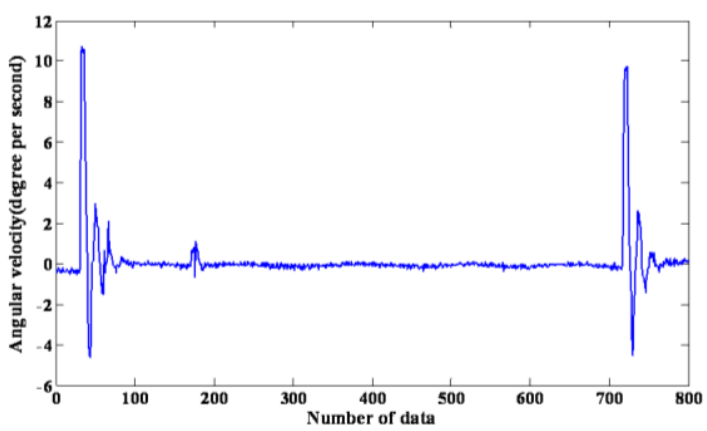

(b)

Fig. 6: (a) The signal corresponding to the applied command from the image processing algorithm to the two degrees of freedom gyro stabilized platform; (b) The output signal from the gyroscope mounted on the yaw axis resulting from the command applied by the image processing algorithm to the two degrees of freedom gyro stabilized platform.

To obtain the transfer function of the two degrees of freedom gyro stabilized platform by using the input and output signals stored during the test, it is enough to specify the input and output of the system identification toolbox in MATLAB and obtain the transfer function corresponding to the given input and output signals.

The transfer function obtained for the two degrees of freedom gyro stabilized platform used in this test was obtained as (1):

$$
\mathrm{Tf}=\frac{3002 \mathrm{~s}^{2}-380.9 \mathrm{~s}+5.896 \mathrm{e}^{04}}{\mathrm{~s}^{4}+69.63 \mathrm{~s}^{3}+2977 \mathrm{~s}^{2}+1353 \mathrm{~s}+5.749 \mathrm{e}^{04}}
$$


Figure 7 shows the step response of the above transfer function.

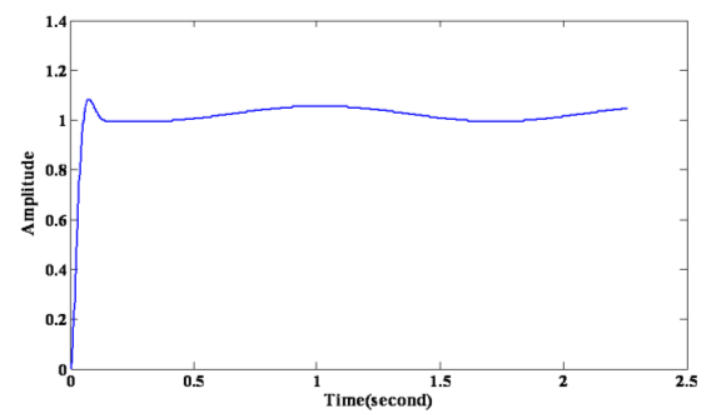

Fig. 7: Step response of the transfer function obtained for the two degrees of freedom gyro stabilized platform.

\section{Comparison between Step Response Obtained from Proposed Method and Step Response Obtained from Simulation}

Figure 8 shows the block diagram of the control system (for the yaw axis) used for obtaining step response of the system.

The step response of the studied two degrees of freedom gyro stabilized platforms in the Simulink was obtained as follows: first, a step signal in the form of angular velocity was applied to the simulated yaw axis structure (Fig. 8). The corresponding output to this input is shown in Fig. 9.

The corresponding response of the studied platform to the step signal obtained experimentally was compared to that obtained from the simulation in Simulink, yielding a difference within the $10 \%$ range. The most important reason can be expressed for this difference in results is the lack of equality in simulations conditions in Simulink with real conditions in laboratory.

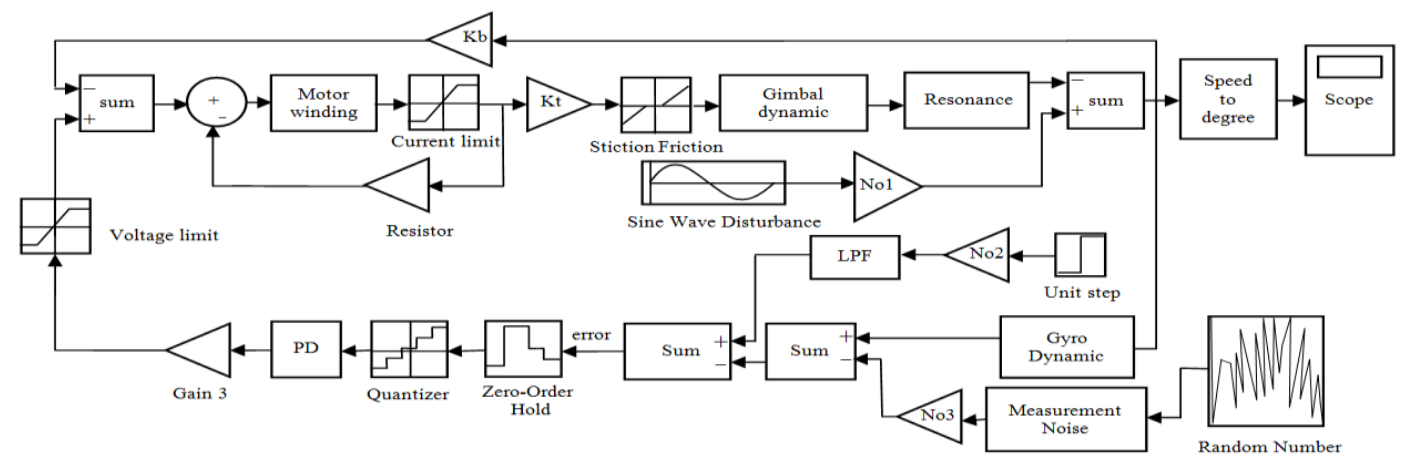

Fig. 8: Control system block diagram at the yaw axis.

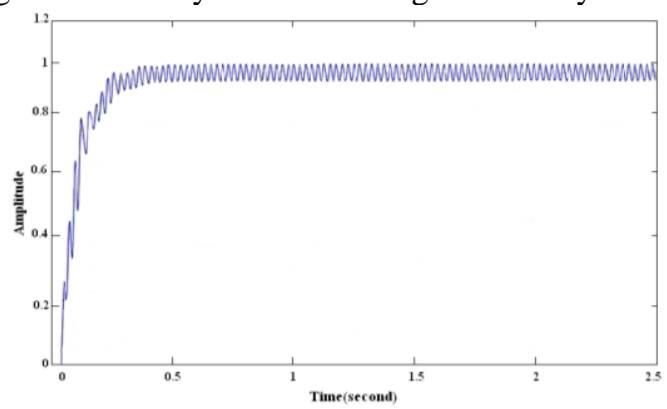

Fig. 9: Step response of the simulated Control system at the yaw axis obtained for the two degrees of freedom gyro stabilized platform. 


\section{Conclusion}

In this article, first an introduction was presented about two degrees of freedom gyro stabilized platforms and their applications in industry, trade, etc. Then, the structure and functioning of two degrees of freedom gyro stabilized platforms as well as components of a two degrees of freedom gyro stabilized platform was discussed. An experimental method was proposed for obtaining the transfer function of a two degrees of freedom gyro stabilized platform by applying a single signal at the input to this platform, storing the corresponding output using a system identification toolbox in MATLAB, and finally, obtaining the transfer function of the studied platform. Also, the step response of the studied platform obtained in the laboratory was compared to that obtained from MATLAB/SIMULINK simulation. The good agreement (within 10\%) observed between these results confirmed the authenticity of the proposed method.

An important aspect in the two degrees of freedom gyro stabilized platforms which must be considered in their design is that these platforms are utilized under different ambient conditions which directly affect the behaviour of the prototype two degrees of freedom gyro stabilized platform. For this reason, further research can be conducted in the following respects: effect of temperature and humidity on the behaviour of the two degrees of freedom gyro stabilized platform and the general performance thereof, and effect of the changes in component behaviour due to environmental/working conditions on the step response and transfer function of these platforms. This research will be conducted in the future.

\section{References}

[1] M. Masten. (2008, Feb). Inertially Stabilized Platforms for Optical Imaging Systems Tracking dynamic targets with mobile sensors. IEEE Trans. Control Systems Magazine. 28(1), pp. 47-65.

[2] P.J. Kennedy and R.L. Kennedy. (2003, Jan). Direct Versus Indirect Line of Sight (LOS) Stabilization. IEEE Trans. Control Systems Technology, 11(1), pp. 3-15.

[3] J.M. Hilkert. (2008, Feb). Inertially Stabilized Platform Technology Concepts and Principles. IEEE Trans. Control Systems Magazine. 28(1), pp. 26-46.

[4] H. Khodadadi, M. Jahed Motlagh and M. Gorji, "Robust control and modeling a 2-DOF Inertial Stabilized Platform," Int. Conf. on Electrical, Control and Computer Engineering, Pahang, Malaysia, 2011, pp. 223-228.

[5] M. Abdoa, A. Toloei, A. Vali and M. Arvan. (2014, Jan). Modeling, Control and Simulation of Cascade Control Servo System for One Axis Gimbal Mechanism. International Journal of Engineering. 27(1), pp. 157-170.

[6] L. Zhi-qiang, Z. Zhi-yong, Z. Qing-kun and F. Da-peng. (2013). Parameter identification of inertially stabilized platforms using current command design. Springer. Vol.20, No. 2, pp. 342-353.

[7] W. Ji, Q. Li, B. Xu, J.j. Tu, D.a. Zhao. (2009). Cascade Servo Control for LOS Stabilization of Opto-electronic Tracking Platform Design and Self-tuning. International Conference on Information and Automation. pp. 1034-1039.

[8] J. Eklånge, "Design and Implementation of a Test Rig for a Gyro Stabilized Camera System," M.S. Thesis, Dept. Electrical. Eng., Linköping Univ., Linköping, Sweden, 2006.

[9] L. Shan-zhong and S. Long-he, "Research on Stabilizing and Tracking Control of Electro-Optical Tracking and Sighting Platform Based on Fuzzy Control," Int. Conf. on Measuring Technology and Mechatronics Automation, Changsha, china, 2010, pp. 175-178. 